\title{
Investigation of pressure distributions for a finite elastohydrodynamic journal bearing lubricated by Ferro fluids with couple stresses
}

\author{
Kihuga Daniel $^{1}$, Kinyanjui Mathew ${ }^{1}$, Kimathi Mark ${ }^{2}$ \\ ${ }^{1}$ Pure and Applied Mathematics Department, Jomo Kenyatta University of Agriculture and Technology, Nairobi, Kenya \\ ${ }^{2}$ Pure and Applied Mathematics Department, Technical University of Kenya, T. U. K, Nairobi, Kenya \\ Email address: \\ Kihugadaniel36@gmail.com (K. Daniel),mathewkiny@gmail.com (K. Mathew),memkimathi@gmail.com (K. Mark)
}

\section{To cite this article:}

Kihuga Daniel, Kinyanjui Mathew, Kimathi Mark. Investigation of Pressure Distributions for a Finite Elastohydrodynamic Journal Bearing Lubricated by Ferro Fluids with Couple Stresses. American Journal of Applied Mathematics. Vol. 2, No. 4, 2014 , pp. 135-140. doi: $10.11648 /$ j.ajam.20140204.14

\begin{abstract}
The performance of finite elastohydrodynamic journal bearings lubricated by ferrofluids with couple stresses has been studied. We derive a Reynolds equation that takes into account magneto elastohydrodynamics, by using the continuity and momentum equations. The equation has been integrated locally across the film thickness and an equation for the pressure gradient obtained as a function of film thickness and total mass flow rate of lubricant, the modified Reynolds equation. The modified Reynolds equation obtained is solved numerically by the finite difference technique since it is highly non-linear. The numerical scheme used is implemented in MATLAB so as to obtain the approximate solutions. The pressure distributions are obtained and the solutions obtained represented in graphs. It is clear from the results that the pressure increases with the increase in magnetism and the couple stress.
\end{abstract}

Keywords: Journal Bearing, Reynolds Equation, Magneto-Elasto-Hydrodynamic Lubrication, Couple Stresses

\section{Introduction}

A bearing is a system of machine elements whose function is to support an applied load by reducing friction between the relatively moving surfaces. Journal bearing is subset of bearings used to support rotating shafts that use the principle of hydrodynamic lubrication. The journal bearing is made up of four main parts as in the Fig 1. This type of bearings is one of the most common forms and is used in a wide variety of machines. When designing such lubricating system, the heat, load and flow rate required for a bearing must be known to properly size the lubricating oil pumps, coolers among other bearing machines. Bearing type and size are based on rotor weight, rotor rotations per minute (RPM), and lubricant characteristics Govindaraj etal (2012).

The characteristics of hydrodynamic journal bearing have been the subject of many researches. Some being directed to the bearing geometry design and others devoted to the study of the lubricant properties. The recent solutions (Nada \& Osman, 2007) that have encouraged the study of the lubricant properties have been used with other parameters being considered in predicting the journal bearing behavior.
These behaviors such as the load carrying capacity, type of lubricant flow region (laminar or turbulent flow), type of lubricant (Newtonian or non-Newtonian), inertia and acceleration effects, and magnetic effect in the case of using ferrofluid (Nada, 2002).

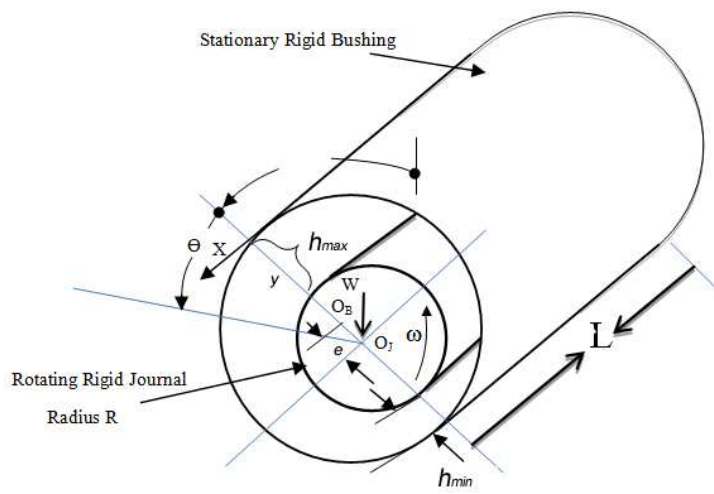

Figure 1. Scheme of the examined bearing

Although many aspects of bearing performance are fully solved, there is still need for further investigations on how to 
improve the bearing performance and its characteristics

\section{Literature Review}

The journal bearing design parameter such as load capacity can be determined from Reynolds equation both analytically and numerically (Hamrock, 1994). Saynatjoki and Holmberg (1982) described a magnetic fluid as not a simple fluid; it is a stable suspension of small particles of ferromagnetic materials in a base fluid. For the purpose of ensuring the colloid stability, a surfactant of polymers (such as oleic acid) is usually introduced into the suspension. This will create around each single particle a coating layer to prevent the agglomeration of the particles by the magnetic field effect or by the molecular attraction. When a magnetic field is applied to the magnetic fluid, each particle experiences a force that depends on the magnetization of the magnetic material of the particles and on the strength of the applied field.

A number of works have applied the micro continuum theory (Stokes, 1966) to investigate the effect of couple stresses on the performance of different types of fluid-film bearings. Based on the micro continuum theory, the problem of lubrication of finite hydrodynamic journal bearing lubricated by magnetic fluids with couple stresses was investigated and by including these couple stresses due to micro structure additives and the magnetic effects due the magnetization of the magnetic fluid, the modified Reynolds equation was obtained to study bearing characteristics. The studies cited above consider the magnetic fluid to behave as a Newtonian fluid but other researches treated the magnetic fluid as non- Newtonian fluid using the power-law model according to Osman (1999), Osman et al (2002) and Osman et al (2003).

Bujurke and Naduvinamani (1990) invested the lubrication of lightly loaded cylinders in combined rolling, sliding, and normal motion with a couple stress fluids as lubricant under cavitation boundary conditions was done. They found that the load capacity and the frictional drag increased as the squeeze velocity increased. Increasing the couple stress parameter enhanced this increase. Presentation on the study of performance of finite journal bearings lubricated with a fluid with couple stresses taking into account the elastic deformation of the liner have been done by Mokhiamer et al (1999). They concluded that the influence of couple stresses on the bearing characteristics was apparently significant.

Naduvinamani et al (2002) investigated the static characteristics of rotor bearing systems lubricated with couple stress fluids and taking into account of surface roughness. Nada and Osman (2007) investigated the static performance of finite hydrodynamic journal bearings lubricated by magnetic fluids with couple stresses and among their conclusion was that the magnetic lubricant gives higher load carrying capacity, higher attitude angle, lower friction coefficient, and higher side leakage. These effects are more significant where the hydrodynamic effects are low, at the lower values of couple stress parameter and eccentricity ratios.

Hydrodynamic lubrication investigations have been the key research studies for many researchers, however in this study, we focus on the velocity and pressure distributions in a magneto-elasto-hydrodynamic lubrication system.

\section{Governing Equations}

The induced magnetic force depends mainly on the applied magnetic field and on the magnetization of the particles magnetic material. The effects of the magnetic force on the pressure of elasto-hydrodynamic journal bearing are also considered studied. The effects of couple stresses on the pressure of elasto-hydrodynamic journal bearing are also considered.

\subsection{Modified Reynolds Equation}

From micro-continuum theory, the general momentum equation of an incompressible fluid with couple stress is given as;

$$
\rho \frac{D \vec{q}}{D t}=-\nabla P+F_{m}+\frac{1}{2} \nabla \times B+\left(\mu-\eta \nabla^{2}\right) \nabla^{2} \vec{q}
$$

In $x$ direction

$$
\rho\left(\frac{\partial u}{\partial t}+u \frac{\partial u}{\partial x}\right)=-\frac{\partial P}{\partial x}+F_{m x}+\mu \frac{\partial^{2} u}{\partial x^{2}}-\eta \frac{\partial^{4} u}{\partial x^{4}}
$$

In $y$ direction

$$
\rho\left(\frac{\partial v}{\partial t}+v \frac{\partial v}{\partial y}\right)=-\frac{\partial P}{\partial y}+F_{m y}+\mu \frac{\partial^{2} v}{\partial y^{2}}-\eta \frac{\partial^{4} v}{\partial y^{4}}
$$

In $z$ direction

$$
\rho\left(\frac{\partial w}{\partial t}+w \frac{\partial w}{\partial z}\right)=-\frac{\partial P}{\partial z}+F_{m z}+\mu \frac{\partial^{2} w}{\partial z^{2}}-\eta \frac{\partial^{4} w}{\partial z^{4}}
$$

Where:

$F_{m x}, F_{m y}$ and $F_{m z}$ are the components of external forces per unit volume. $\rho\left(\frac{\partial u}{\partial t}+u \frac{\partial u}{\partial x}\right), \rho\left(\frac{\partial v}{\partial t}+v \frac{\partial v}{\partial y}\right)$ and $\rho\left(\frac{\partial w}{\partial t}+w \frac{\partial w}{\partial z}\right)$ are the three components of acceleration of the fluid.

Equations (3.2) to (3.4) contain the four unknowns' $u, v, w$ and $p$. the fourth equation is supplied by the continuity equation:

$$
\frac{\partial \rho u}{\partial x}+\frac{\partial \rho v}{\partial y}+\frac{\partial \rho w}{\partial z}=0
$$

Applying the assumptions below;

1. The lubricant is assumed to be Newtonian fluid.

2. The flow is laminar; consequently, neither vortex flow nor turbulence is occurring anywhere in the flow.

3. The lubricant is assumed incompressible; i.e. its density is constant. 
4. The curvature of the fluid film is neglected since the film thickness in y-direction is very thin compared with the span in $\mathrm{x}$ and $\mathrm{z}$-directions. Thus, no gradient of the applied magnetic field across the fluid film, no magnetic force in y-direction and thus no pressure gradient in this direction $\frac{\partial P}{\partial y}=0$

5. The fluid flow is steady. Implying that

$$
\frac{\partial u}{\partial t}=\frac{\partial y}{\partial t}=\frac{\partial w}{\partial t}=0
$$

6. The fluid inertia force is neglected compared to the viscous force and the induced magnetic force

7. No slipping at the bearing surface

8. Except $\frac{\partial u}{\partial y}$ and $\frac{\partial w}{\partial y}$, all other velocity gradients are considered negligible.

9. No heat conducted to or from the fluid film to surfaces (adiabatic case).

On application of the above assumptions the equations 3.2, 3.3 and 3.4 reduces to;

$$
\begin{gathered}
\frac{\partial P}{\partial x}=F_{m x}+\mu \frac{\partial^{2} u}{\partial y^{2}}-\eta \frac{\partial^{4} u}{\partial y^{4}} \\
\frac{\partial P}{\partial y}=0 \\
\frac{\partial P}{\partial z}=F_{m z}+\mu \frac{\partial^{2} w}{\partial y^{2}}-\eta \frac{\partial^{4} w}{\partial y^{4}}
\end{gathered}
$$

Where $F_{m x}$ and $F_{m z}$ are the magnetic force components in circumferential and axial directions respectively. The boundary conditions can be stated as below;

$$
\begin{array}{r}
\text { At } y=0 \quad u=0 \quad v=0 \quad w=0 \\
\frac{\partial^{2} u}{\partial y^{2}}=0 \quad, \frac{\partial^{2} w}{\partial y^{2}}=0 \\
\text { At } y=\frac{h}{2} \quad u=\frac{\omega \Re}{h}, v=0 \quad w=0 \\
\frac{\partial u}{\partial y}=0 \quad, \frac{\partial w}{\partial y}=0
\end{array}
$$

At $y=h \quad u=\omega \Re, v=0 \quad w=0$

$$
\frac{\partial^{2} u}{\partial y^{2}}=0 \quad, \frac{\partial^{2} w}{\partial y^{2}}=0
$$

Equations (3.9), (3.11) and (3.13) are the no-slip boundary conditions. Equations (3.10), (3.12) and (3.14) result from the couple stress and vanish at the solid surfaces (Nada \& Osman, 2007). This is due to the resistance of the solid surfaces for the rotation motion of the additive particles.

By solving equations (3.6) and (3.8) and using the above boundary conditions, the velocity profiles in circumferential and axial directions can be obtained (Nada \& Osman, 2007). These velocities are substituted in the following integrated continuity equation across the fluid film thickness for an incompressible fluid flow and then solved to give the specific Reynolds equation.

$$
\int_{0}^{h}\left(\frac{\partial u}{\partial x}+\frac{\partial v}{\partial y}+\frac{\partial w}{\partial z}\right) d y=0
$$

The generalized pressure equation is obtained in the form;

$$
\begin{aligned}
& \frac{\partial}{\partial x}\left(\frac{g(h, l)}{\mu} \frac{\partial P}{\partial x}\right)+\frac{\partial}{\partial z}\left(\frac{g(h, l)}{\mu} \frac{\partial P}{\partial z}\right)=6 \omega \Re \frac{\partial h}{\partial x} \\
& +\frac{\partial}{\partial x}\left(\frac{g(h, l)}{\mu} F_{m x}\right)+\frac{\partial}{\partial z}\left(\frac{g(h, l)}{\mu} F_{m z}\right)
\end{aligned}
$$

Where;

$$
g(h, l)=h^{3}-12 l^{2} h+24 l^{3} \tanh \left(\frac{h}{2 l}\right)
$$

Abdo, 2009 stated that the components of magnetic force ( $F_{m x}$ and $F_{m z}$ ) can be obtained in the $\mathrm{x}$ and $\mathrm{z}$ directions respectively as below;

$$
\begin{aligned}
& F_{m x}=\mu_{0} X_{m} h_{m} \frac{\partial h_{m}}{\partial x} \\
& F_{m z}=\mu_{0} X_{m} h_{m} \frac{\partial h_{m}}{\partial z}
\end{aligned}
$$

Substituting equations (3.17) and (3.18) in the Reynolds equation (3.16) above we obtain;

$$
\begin{gathered}
\frac{\partial}{\partial x}\left(\frac{g(h, l)}{\mu} \frac{\partial P}{\partial x}\right)+\frac{\partial}{\partial z}\left(\frac{g(h, l)}{\mu} \frac{\partial P}{\partial z}\right)=6 \omega \Re \frac{\partial h}{\partial x}+ \\
\frac{\partial}{\partial x}\left(\frac{g(h, l)}{\mu} \mu_{0} X_{m} h_{m} \frac{\partial h_{m}}{\partial x}\right)+\frac{\partial}{\partial z}\left(\frac{g(h, l)}{\mu} \mu_{0} X_{m} h_{m} \frac{\partial h_{m}}{\partial z}\right)
\end{gathered}
$$

\subsection{Non-Dimensionalization}

The subject of dimensional analysis considers how to determine the required the set of scales for any given problem. This is a process that starts with selecting a suitable scale against which all dimensions in a given physical model are scaled. This process aims at ensuring that the results obtained are applicable to other geometrically similar configurations under similar set of flow conditions.

The non-dimensional parameters used are defined as below:

$$
\begin{gathered}
x=R \theta \quad z=L_{b} Z \quad e=C \varepsilon \quad h=C H \quad l=C L \\
L_{b}=2 R \beta \quad h_{m}=h_{m o} H \quad \text { And } P=\frac{\lambda_{0} \omega P^{*}}{(C / R)^{2}}
\end{gathered}
$$

Using these non-dimensional parameters, the modified Reynolds equation (3.25) becomes; 


$$
\begin{array}{r}
\frac{\partial}{\partial \theta}\left(G(H, L) \frac{\partial P^{*}}{\partial \theta}\right)+\frac{1}{4 \beta^{2}} \frac{\partial}{\partial z}\left(G(H, L) \frac{\partial P^{*}}{\partial z}\right)=6 \frac{\partial H}{\partial \theta} \\
+4 \beta^{2} \alpha \frac{\partial}{\partial \theta}\left(G(H, L) H_{m} \frac{\partial H_{m}}{\partial \theta}\right)+\alpha \frac{\partial}{\partial z}\left(G(H, L) H_{m} \frac{\partial H_{m}}{\partial z}\right)
\end{array}
$$

Where

$$
G(H, L)=H^{3}-12 L^{2} H+24 L^{3} \tanh \left(\frac{H}{2 L}\right)
$$

$\alpha=\frac{\left(h_{m o}\right)^{2} \lambda_{0} X_{m} C^{2}}{\mu_{0} \omega\left(L_{b}\right)^{2}}$

It is noted that, as the value of $(L)$ tend to zero, equation (3.20) reduced to the Newtonian magnetic lubricant case and the effect of couple stresses vanishes.

$$
\begin{aligned}
& \frac{\partial}{\partial \theta}\left(H^{3} \frac{\partial P^{*}}{\partial \theta}\right)+\frac{1}{4 \beta^{2}} \frac{\partial}{\partial z}\left(H^{3} \frac{\partial P^{*}}{\partial z}\right)=6 \frac{\partial H}{\partial \theta} \\
& +4 \beta^{2} \alpha \frac{\partial}{\partial \theta}\left(H^{3} H_{m} \frac{\partial H_{m}}{\partial \theta}\right)+\alpha \frac{\partial}{\partial z}\left(H^{3} H_{m} \frac{\partial H_{m}}{\partial z}\right)
\end{aligned}
$$

\subsection{Finite Difference Technique}

\subsubsection{Introduction}

The performance of the bearing in this study has been predicted assuming adiabatic conditions with magnetic fluid lubricant with couple stress. The effect of magnetic force coefficient, couple stress parameter and length to diameter ratio and pressure distribution has been numerically investigated

\subsubsection{The Reynolds Equation in Finite Difference}

Back to the specific non-dimensional Reynolds equation (3.21)

$$
\frac{\partial}{\partial \theta}\left(H^{3} \frac{\partial P^{*}}{\partial \theta}\right)+\frac{1}{4 \beta^{2}} \frac{\partial}{\partial z}\left(H^{3} \frac{\partial P^{*}}{\partial z}\right)=6 \frac{\partial H}{\partial \theta}+
$$

$$
4 \beta^{2} \alpha \frac{\partial}{\partial \theta}\left(H^{3} H_{m} \frac{\partial H_{m}}{\partial \theta}\right)+\alpha \frac{\partial}{\partial z}\left(H^{3} H_{m} \frac{\partial H_{m}}{\partial z}\right)
$$

Considering the bearing geometry and the magnetic field model, the film thickness $H$ depends totally on the change of the angle $\theta$ and hence the derivatives of $H$ with respect to $\theta$ is retained while those of $\mathrm{Hm}$ with respect to $\theta$ vanishes, also the derivatives of $H$ with respect to $Z$ disappears while those of $\mathrm{Hm}$ with respect to $Z$ is retained thus the equation reduces to;

$$
\begin{aligned}
3 H^{2} \frac{\partial H}{\partial \theta} \cdot \frac{\partial P^{*}}{\partial \theta}+H^{3} \frac{\partial^{2} P^{*}}{\partial \theta^{2}}+\frac{1}{4 \beta^{2}} H^{3} \frac{\partial^{2} P^{*}}{\partial Z^{2}}=6 \frac{\partial H}{\partial \theta} \\
+\alpha\left\{H^{3}\left(\frac{\partial H_{m}}{\partial z}\right)^{2}+H^{3} H_{m} \frac{\partial^{2} H_{m}}{\partial z^{2}}\right\}
\end{aligned}
$$

Applying the finite difference technique and using the central difference method, the modified Reynolds equation gets discretized to;

$$
\begin{aligned}
P_{i, j}^{*}\left[\frac{2\left(H_{i, j}\right)^{3}}{(\Delta \theta)^{2}}+\frac{1}{2 \beta^{2}} \frac{\left(H_{i, j}\right)^{3}}{(\Delta Z)^{2}}\right] & =-3\left(H_{i, j}\right)^{2}\left[\frac{H_{i+1, j}-H_{i-1, j}}{2 \Delta \theta}\right] \times\left[\frac{P_{i+1, j}-P_{i-1, j}}{2 \Delta \theta}\right] \\
& -\left(H_{i, j}\right)^{3}\left[\frac{P_{i+1, j}-P_{i-1, j}}{(\Delta \theta)^{2}}\right]-\frac{\left(H_{i, j}\right)^{3}}{4 \beta^{2}}\left[\frac{P_{i, j+1}-P_{i, j-1}}{\left.(\Delta Z)^{2}\right]}\right. \\
& +3\left[\frac{\left.H_{i+1, j}-H_{i-1, j}\right]+\alpha\left(H_{i, j}\right)^{3}\left\{\left(\frac{H m_{i, j+1}-H m_{i, j-1}}{2 \Delta Z}\right)^{2}\right\}}{\Delta \theta}\right] \\
& +\alpha H m_{i, j}\left(\frac{H m_{i, j+1}-H m_{i, j-1}+2 H m_{i, j}}{(\Delta Z)^{2}}\right)
\end{aligned}
$$

\section{Results and Discussion}

The journal bearing used is as in the Figure 1. It has two components, one representing the shaft and the other the bearing. The system is supplied by lubricant fluid through openings which emerge in an axial groove.

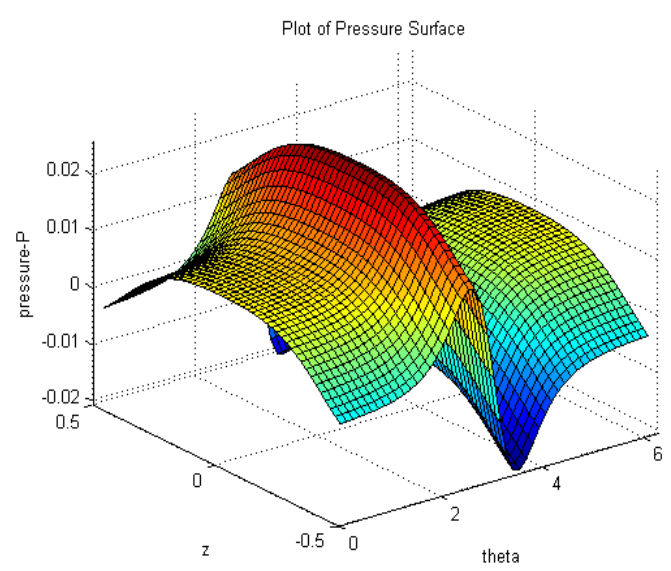

Figure 2. Plot surface of pressure with theta and $z$ 
The results as in Figure 2 give the dimensionless pressure distribution in circumferential and axial directions with eccentricity ratio for different values of the couple stress parameter. It represents a surface of pressure against both circumferential and axial directions. The results are obtained for constant eccentricity ratio e, length to diameter ratio $\beta$ of 1.0 and varied couple stress parameter $L$ of $0.0,0.2$ and 0.4 (Abdo, 2009) where $\mathrm{L}=0.0$ is the Newtonian lubricant case. The results are also determined for magnetic coefficient $\alpha=0.0$ which is the non-magnetic lubricant case and $\alpha=2.0$ to 8.0 the magnetic lubricant case (Nada $\&$ Osman, 2007). The pressure distribution in the circumferential direction at the bearing mid-plane (bearing centerline) for different values of couple stress parameter and a constant value of magnetic coefficient are shown in Fig 3 for eccentricity ratio $\mathrm{e}=0.6$.

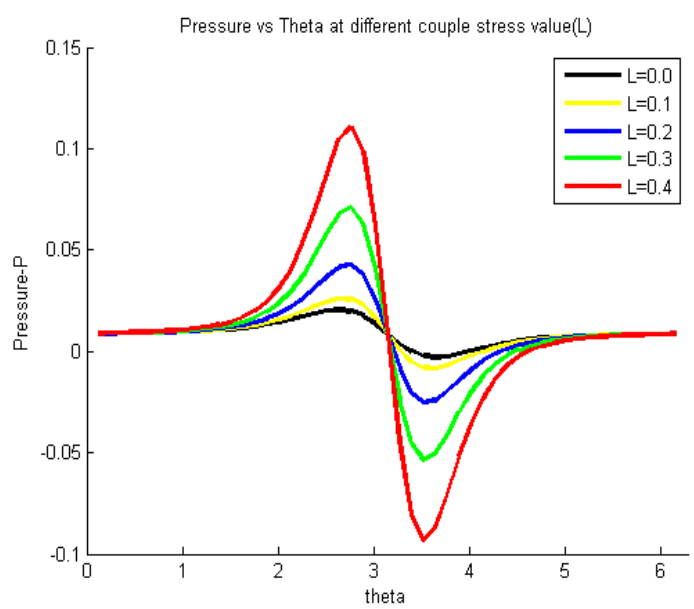

Figure 3. Plot of pressure against theta with increasing couple stress

It is clearly shown in Figure 3 that for magnetic lubricant, there is an increase of the pressure with increasing $\mathrm{L}$ at a constant eccentricity ratio used. This increase is more pronounced as we increase the value of the couple stress parameter. Thus, the pressure is increasing as the value of the couple stress parameter increases.

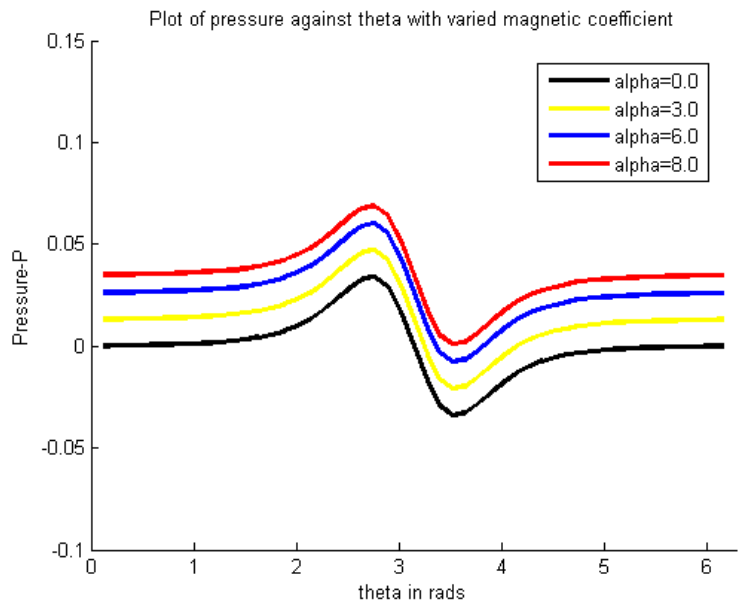

Figure 4. Plot surface of pressure against theta with varied magnetic coefficient
Considering the magnetic effect using the magnetic coefficient, we vary the value of the magnetic coefficient to see the effect. This is evident clearly as from Figure 4 . There is a large increase of the pressure as the value of the magnetic coefficient increases. The maximum pressure is shifted to the angle $\theta=0.5 \pi$. Due to symmetry from Fig 2 it is clear that the pressure increases in magnitude in the negative way after another $0.5 \pi$ mark angle. Effect of alpha is also determined at a constant value of the eccentricity ratio. At this value of eccentricity ratio, there are two maximum pressure points as shown in Fig land also according to Abdo, 2009. The first at $\theta=0.5 \pi$ due to the magnetic effect and value of the couple stress parameter $L$ and the other at nearly $\theta=\pi$ due to the hydrodynamic effect, its value depends mainly on the couple stress parameter L.

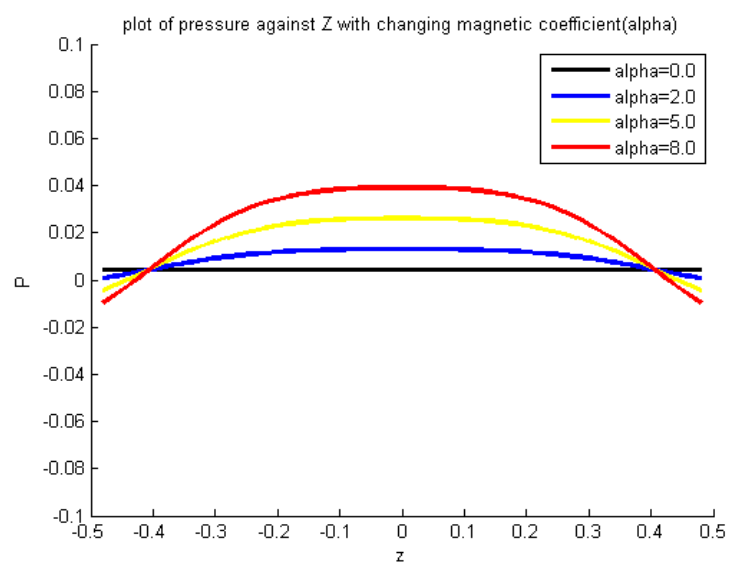

Figure 5. Plot surface of pressure with theta and $z$

Figure 5 represents the plot of pressure against the length of the bearing for different couple stress parameter for magnetic lubricant ( $\alpha=2.0$ ). It is clear that as we increase the value of the magnetic coefficient the pressure also increases. The magnetic contribution is more along the length of the bearing and it is highest at the middle of the bearing as evident in Figure 5. Some symmetry is seen at the center of the bearing that is at $Z=0.0$. All this is being analyzed considering the same eccentricity ratio being held a constant, it can also be seen that the pressure of the bearing lubricated with couple stress fluid increases with increasing the couple stress parameter. Bearings pressure with magnetic lubricant and couple stress are greater than that with nonmagnetic lubricant without the couple stress.

\section{Conclusion}

The results concluded that the magnetic lubrication gives higher pressure distribution. Based on the micro-continuum theory (Stokes, 1966), and by taking into account the couple stresses due to the microstructure additives, the effects of couple stresses on the performance of a finite elastohydrodynamic journal bearings were studied. The results have shown that lubricants with couple stresses compared with Newtonian lubricants increase the pressure. Thus it can be concluded that fluids with couple stresses are 
better than Newtonian fluids. Considering the magnetism of the fluid, it is clear that ferrofluids gives higher pressure to the bearing hence improving on the efficiency of the system. Therefore, it can be concluded that lubricants with additives are better than the common lubricants used. The results are thereafter expected to provide to the engineers useful information to design machine elements and bearing systems with a higher life expectancy and efficiency.

\section{Nomenclature}

C Bearing clearance, $m$

$e$ Eccentricity of the journal center, $m$

$\mathcal{E}$ Eccentricity ratio $(\varepsilon=e / C)$

$F_{m}$ Unit volume value of the induced magnetic force, Tesla

$F_{m x} \quad$ Magnetic force in $x$ direction (circumferential direction)

$F_{m z} \quad$ Magnetic force in $z$ direction (axial direction)

$M_{g}$ Magnetization of the magnetic material, $\mathrm{Wbm}^{-2}$

$M_{g s} \quad$ Saturation value of magnetization

$h$ Lubricant film thickness, $m$

$H \quad$ Non-dimensional film thickness $(H=h / C)$

$u_{i} \quad$ Fluid velocity vector in $\mathrm{x}, \mathrm{y}$ and $\mathrm{z}$ directions

$u, v, w$ Components of velocity vector $\vec{q} \quad\left(m s^{-1}\right)$

$x, y, z \quad$ Cartesian coordinates

$\theta$ Angular coordinate $(x / R)$

$R \quad$ Bearing or journal radius

$Z$ Non-dimensional axial distance $\left(Z=z / L_{b}\right)$

$h_{\max }$ Maximum film thickness, $m$

$h_{\min } \quad$ Minimum film thickness, $m$

$h_{m} \quad$ Magnetic field intensity, $\mathrm{Wbm}^{-2}$

$h_{m 0} \quad$ Characteristic value of magnetic field intensity

$H_{m} \quad$ Non-dimensional magnetic field intensity

$P$ Lubricant pressure, $N / \mathrm{m}^{2}$

$P^{*} \quad$ Non-dimensional pressure $P^{*}=\frac{P(C / R)^{2}}{\mu_{0} \omega}$

$\alpha \quad$ Magnetic force coefficient $\alpha=\frac{\left(h_{m o}\right)^{2} \lambda_{o} X_{m} C^{2}}{\mu_{0} \omega\left(L_{b}\right)^{2}}$

$\rho$ Fluid density, $\mathrm{Kg} / \mathrm{m}^{3}$

$\mu$ Fluid viscosity, pa-s

$\mu^{*}$ Non-dimensional viscosity

$l$ Couple stress parameter

$L \quad$ Non-dimensional couple stress parameter $(L=l / C)$ $\omega$ Angular speed, $\mathrm{rad} / \mathrm{s}$

$\eta$ Material constant responsible for the couple stress property
$\vec{\nabla} \quad$ Gradient operator $i \frac{\partial}{\partial x}+j \frac{\partial}{\partial y}+k \frac{\partial}{\partial z}$
$\vec{F} \quad$ Body forces vector in $\mathrm{x}, \mathrm{y}$ and $\mathrm{z}$ directions

\section{References}

[1] Abdo, H. S. M. (2009). Thermal effects on hydrodynamic journal bearing lubricated by magnetic fluids with couple stresses.

[2] Bujurke N. M. \& Naduvinamani N. B., (1990). The lubrication of lightly loaded cylinders in combined rolling, sliding and normal with couple stress fluid, Int. Journal Mech. Science.

[3] Cowley, m. D., \& Rosensweig, r. E. (April 1967). The interfacial stability of ferromagnetic fluid. Journal of fluid mech, 671-688.

[4] Govidaraj, R. \& Satishkumar V. M. (2012). Design of journal bearing test rig, Sweden blekinge Tekniska Hogskola.

[5] Mokhiamer U. M., Crosby W. A. \&El-Gamal H. A., (1999). A study of a journal bearing lubricated by fluids with couple stress considering the elasticity of the liner wear.

[6] Nada, g. S. (2002). Static and dynamic characteristics of magnetized journal bearings lubricated with ferrofluid. PhD thesis in mechanical engineering, faculty of engineering. Cairo, Egypt: Cairo University.

[7] Nada, g. S., \& Osman, t. A. (2007). Static performance of finite hydrodynamic journal bearings lubricated by magnetic fluids with couple stresses. Tribology letters, 261-268.

[8] Naduvinamani N. B., Hiremat P. S. \& Gurubasavaraj G. (2002). Effects of surface roughness on the static characteristics of rotor bearing with couple stress fluid, Comput Struct.

[9] Osman T. A., (1999). Static characteristics of hydrodynamic magnetic bearings working by Non-Newtonian Ferrofluid, Journal of Engineering and Applied science, Cairo University, $521-536$

[10] Osman T. A., Nada G. S. \& Safar Z. S., (2003). Different magnetic models in the design of hydrodynamic journal bearings lubricated with Non-Newtonian Ferrofluid, Tribology letters, 211-223

[11] Stokes, V. K. (1966). Couple stresses in fluids. Phys. Fluids, 1709-1715

[12] Saynatjoki, M, and Holmberg K., (1982) Magnetic fluids in sealing and lubrication-a state of art review. Finland: Technical Research Center of Finland. 